\title{
UTILIZACIÓN DE Atriplex canescens Y Opuntia ficus indica EN LA ALIMENTACIÓN DE CABRAS LACTANTES DURANTE LA SEQUÍA ${ }^{1}$
}

\author{
Jorge Urrutia-Morales², Héctor Guillermo Gámez-Vázquez², Sergio Beltrán-López ${ }^{3}$, Marta Olivia Díaz-Gómez ${ }^{3}$
}

\begin{abstract}
RESUMEN
Utilización de Atriplex canescens y Opuntia ficus indica en la alimentación de cabras lactantes durante la sequía. El objetivo del presente estudio fue evaluar el efecto del Atriplex (Atriplex canescens) y nopal (Opuntia ficus indica) en la alimentación de cabras lactantes y la producción de leche durante la sequía. Durante los meses secos, entre mayo y agosto de 2005 y entre mayo y junio de 2006, se realizaron tres experimentos en la región semiárida de San Luis Potosí, México. En el primero, se probaron dos tratamientos: $\mathrm{CO})$ mantenidas en confinamiento y alimentación controlada $(\mathrm{n}=10)$ y AT) mantenidas en pastoreo con Atriplex $(\mathrm{n}=10)$. En el segundo se aplicaron dos tratamientos: AT) Atriplex $(\mathrm{n}=5)$ y NP) Atriplex más nopal $(\mathrm{n}=5)$. En el tercero tres tratamientos: AT) Atriplex ( $\mathrm{n}=5), \mathrm{NP}-1,0)$ Atriplex más $1,0 \%$ de nopal $(\mathrm{n}=4)$ y NP-1,5) Atriplex más $1,5 \%$ de nopal $(\mathrm{n}=5)$. En el primer experimento las cabras mantuvieron su peso, pero la producción de leche se redujo al 30\% bajo confinamiento y menos del 8,0\% en Atriplex al final del experimento. En el segundo, las cabras perdieron peso, a pesar de que la producción inicial de leche fue menor de $300 \mathrm{~g} / \mathrm{d}$. Las alimentadas con Atriplex redujeron su producción a casi la mitad de la producción inicial, mientras que la inclusión de nopal mantuvo la producción relativamente estable. En el tercer experimento, las cabras alimentadas con Atriplex mantuvieron el peso corporal, pero después de siete semanas la producción de leche fue del $25 \%$ de la producción inicial, a pesar de que esta fue de apenas 300 $\mathrm{g}$ diarios. En cambio, en las cabras suplementadas con nopal, la producción sólo se redujo al 45 y $64 \%$ de la producción inicial. Estos resultados son importantes para los caprinocultores de la región semiárida de México, donde las cabras podrían mantener una buena condición corporal, además de una producción de 150 a $250 \mathrm{~g}$ diarios de leche durante la época crítica utilizando Atriplex y nopal.
\end{abstract}

Palabras clave: producción de leche, Atriplex, nopal.

\begin{abstract}
Use of Atriplex canescens and Opuntia ficus indica as feed for lactating goats during drought. The aim of this study was to evaluate the effect of saltbush (Atriplex canescens) and prickly pear (Opuntia ficus indica) in the feeding of goats and milk production during drought. During the dry season, between May and August 2005 and between May and June 2006, three experiments were conducted in the semiarid region of San Luis Potosi, Mexico. In the first, two treatments were tested: $\mathrm{CO}$ ) kept in confinement and controlled feeding $(\mathrm{n}=10)$ and AT) kept in grazing saltbush $(\mathrm{n}=10)$. In the second, two treatments were applied: AT) Atriplex $(\mathrm{n}=5)$ and NP) Atriplex plus cactus $(\mathrm{n}=5)$. In the third, three treatments were applied: AT) Atriplex $(\mathrm{n}=5)$, NP-1,0) Atriplex plus $1,0 \%$ of cactus $(n=4)$ and NP-1,5) $1,5 \%$ Atriplex plus $1.5 \%$ of cactus $(n=5)$. In the first experiment, goats maintained their weight, but milk production was reduced to about $30 \%$ in $\mathrm{CO}$ and less than $8,0 \%$ in TA at the end of the experiment. In the second, goats lost weight, despite the initial milk production was less than $300 \mathrm{~g} / \mathrm{d}$. The goats fed with Atriplex reduced their production to almost half of the initial production, while the inclusion of cactus maintained production relatively stable. In the third experiment, the goats fed with Atriplex maintained their body weight, but after seven weeks milk production was only $25 \%$ of the initial production, even though it was only $300 \mathrm{~g}$ daily. In contrast, in the cactus supplemented goats, production tended to decrease in a lesser extent, reaching 45 and $64 \%$ of the initial production. These results are important for goat producers in the semiarid region of Mexico, where the goats could maintain good body condition, as well a production of 150 to $250 \mathrm{~g}$ of milk daily during the critical season using saltbush and cactus.
\end{abstract}

Keywords: milk production, Atriplex, cactus pad.

\footnotetext{
Recibido: 21 de octubre, 2013. Aceptado: 30 de junio, 2014. Este trabajo forma parte del proyecto "Evaluación del potencial del chamizo para la producción de leche de cabra", financiado por la Fundación Produce de San Luis Potosí A.C.

2 Campo Experimental San Luis, Instituto Nacional de Investigaciones Forestales, Agrícolas y Pecuarias. Carretera San Luis-Matehuala Km 14.5 Ejido Palma de la Cruz, 78430, Soledad de Graciano Sánchez, San Luis Potosí, México. jorurrmo@hotmail.com, gamez.hector@inifap.gob.mx 3 Facultad de Agronomía, Universidad Autónoma de San Luis Potosí. Carretera San Luis-Matehuala Km 14.5, Ejido Palma de la Cruz, 78430, Soledad de Graciano Sánchez, San Luis Potosí, México.belopse@gmail.com,mdiaz@uaslp.mx
} 


\section{INTRODUCCIÓN}

La caprinocultura extensiva es uno de los sistemas de producción pecuaria predominantes en las regiones árida y semiárida de México. La alimentación de los animales se basa en el forraje pastoreado en los pastizales naturales. La escaza precipitación pluvial, concentrada en los meses de verano, ocasiona que la productividad de los pastizales sea marcadamente estacional, lo cual origina que la calidad y disponibilidad de forraje sean altas en los meses de verano y otoño, y bajas el resto del año. En estas condiciones, las cabras experimentan estados de alimentación deficiente en los períodos de estiaje de invierno y primavera (Echavarría et al., 2006), lo que se refleja en pérdidas de peso, pobre desempeño reproductivo y baja producción general (Castillo et al., 1989; Morlán et al., 2005; Olhagaray, 2005). Para mantener una producción sostenida se requiere de alimentos de bajo costo y fácil adquisición para suplementar al ganado en la época de estiaje.

La literatura proporciona información sobre diversos recursos forrajeros que pueden ser producidos en condiciones semiáridas, que brinda los nutrientes necesarios para mejorar el estado nutricional de los animales, entre los que destacan las arbustivas forrajeras (Ramírez et al., 2006; Guerrero et al., 2010). Atriplex canescens es una arbustiva siempre verde distribuida en la mayor parte del norte de México y sur de Estados Unidos. Sus hojas contienen cantidades de proteína superiores a la de muchos forrajes de uso común (Urrutia et al., 1989; Guerrero et al., 2010) y son habitualmente consumidas por las cabras en pastoreo. A pesar de ello, la información disponible sobre su uso es limitada. El follaje de Atriplex se caracteriza por su su alto contenido en sal y de proteína (desde 12 a 17\%) (Urrutia et al., 1989; Guerrero et al., 2010). Esta última característica limita el consumo por los animales, en especial cuando Atriplex constituye la única o principal fuente de nutrientes (Ben Salem et al., 2010). Para contrarrestar el elevado consumo de sal, los animales incrementan el consumo de agua, lo que les permite eliminar el excedente de sal. Sin embargo, en las regiones semiáridas la disponibilidad de agua fresca es limitada y generalmente presenta contenidos de moderados a altos de sal.

Recientemente se ha evaluado el uso de pencas de nopal (Opuntia spp.) como complemento alimenticio de dietas basadas en forraje de Atriplex. El nopal es una planta suculenta nativa de México, tolerante a la sequía y muy eficiente en el uso de agua, que se caracteriza por su bajo contenido de materia seca $(<15 \%)$, nitrógeno y fibra, pero alto contenido de azúcares (Nefzaoui y Ben Salem, 2002). El uso combinado de Atriplex y nopal presenta diversas ventajas. El agua del nopal reduce la necesidad de agua de bebida y contribuye con la excreción de las sales contenidas en Atriplex, mientras que el contenido de azúcares solubles del nopal mejora el aprovechamiento del nitrógeno soluble de Atriplex para la síntesis de proteína microbiana (Nefzaoui y Ben Salem, 2002; Ben Salem et al., 2005).

En corderos alimentados con Atriplex la suplementación con nopal mejoró la ganancia de peso (Ben Salem et al., 2005), a pesar de que los corderos que recibieron nopal redujeron el tiempo que invirtieron en el ramoneo de Atriplex, lo que indica que el uso conjunto de nopal y arbustivas aumenta la eficiencia alimenticia, al mejorar el balance nitrógeno:energía (Ben Salem et al., 2005). Además la digestibilidad de la materia orgánica (MO) se incrementó, así como la retención de $\mathrm{N}$, lo que ayuda a explicar el mejor comportamiento de los corderos.

A pesar de lo anterior, poco se ha reportado sobre el potencial del Atriplex como principal fuente de nutrientes en la producción de leche de cabras, ni sobre el uso de nopal como suplemento de cabras lecheras mantenidas en pastizales semiáridos durante la estación de sequía. El objetivo del presente estudio fue explorar el uso de Atriplex como principal fuente de alimento para cabras lactantes y evaluar el efecto del uso combinado de Atriplex y nopal sobre el comportamiento de la lactancia en cabras durante la sequía.

\section{MATERIAL Y MÉTODOS}

Condiciones experimentales. Este estudio se realizó en el Campo Experimental San Luis del Instituto Nacional de Investigaciones Forestales, Agrícolas y Pecuarias, ubicado en Soledad, San Luis Potosí, México. El sitio está localizado a $22^{\circ} 14^{\prime} 03^{\prime \prime} \mathrm{N}$, $100^{\circ} 53$ ' 11" O y $1835 \mathrm{msnm}$. El clima predominante es semiárido con una temperatura media anual de 17,6 ${ }^{\circ} \mathrm{C}$ y precipitación media anual de $335 \mathrm{~mm}$ distribuida en verano y otoño (Medina et al., 2005). El trabajo experimental se realizó en los meses de mayo a agosto de 2005 y de mayo a junio de 2006. 
Forraje utilizado. Se utilizó una plantación de Atriplex (A. canescens) establecida en dos hectáreas a una densidad de 1250 plantas/ha. Esta plantación permaneció sin uso durante los primeros cuatro años, y después fue pastoreada por cabras cada dos años, en los meses de marzo y abril, incluyendo el año previo al presente estudio. En la plantación, aparte de Atriplex, se desarrollaron de forma espontánea plantas de la arbustiva mariola (Parthenium incanum) y de zacate buffel (Cenchrus ciliaris L.); sin embargo, las únicas plantas verdes presentes durante los experimentos eran las de Atriplex, mientras que otras arbustivas, hierbas anuales y zacates estaban secos y en cantidades marginales.

Como complemento, se utilizaron pencas de nopal sin espinas (O. ficus indica) de una plantación de cuatro años de edad manejada en condiciones de riego. Se cosecharon pencas de menos de un año ubicadas en la parte superior de la planta, las cuales se picaron antes de ofrecerlas a los animales.

Estimación de la biomasa de Atriplex. Para estimar la cantidad de biomasa disponible de Atriplex en la plantación se tomaron medidas de altura (h), diámetro máximo de la planta (d) y número de ramas (n) de quince plantas escogidas al azar. Las medidas se introdujeron en la ecuación previamente diseñada y validada por Beltrán (1993): y=1973,31 + 1453,91(h) $+360,97(\mathrm{~d})+390,05(\mathrm{n})$.

Análisis de laboratorio. Se tomaron muestras de seis plantas de Atriplex escogidas al azar. En cada una se recolectaron las partes consumibles por las cabras (hojas y tallos tiernos), Las muestras de nopal se tomaron de seis plantas elegidas al azar, colectando las pencas ubicadas en la parte superior de la planta. Las muestras de Atriplex y nopal se enviaron al laboratorio para determinar el contenido de materia seca (MS), proteína cruda (PC) (AOAC, 2000), fibra ácido detergente (FAD) (Van Soest et al., 1991) y digestibilidad in vitro de la materia seca (DMS) (Vanzant et al., 1998).

\section{Animales y diseño experimental}

Se realizaron tres experimentos con cabras adultas de la raza Nubia, lactantes y no gestantes, durante los meses secos, entre mayo y agosto de 2005 y entre mayo y junio de 2006. En el primer experimento se compararon dos sistemas de alimentación para evaluar el potencial de Atriplex como principal fuente de nutrientes para producción de leche en época seca del año: AT) mantenidas en una plantación de Atriplex y CO) mantenidas en confinamiento y alimentación controlada (tratamiento control). En el segundo se aplicaron dos tratamientos para evaluar el uso de la penca de nopal como suplemento en cabras lactantes alimentadas con Atriplex: AT) mantenidas en una plantación de Atriplex y NP) similar a AT más nopal $(0,8 \%$ del peso vivo (PV) en base seca (BS). En el tercero se aplicaron tres tratamientos para evaluar el uso de niveles crecientes de penca de nopal como suplemento en cabras lactantes alimentadas con Atriplex, bajo el supuesto de que el mayor consumo de agua y azúcares solubles contenidos en el nopal promoverían un mejor aprovechamiento del forraje de Atriplex, lo que se reflejaría en mejor respuesta animal: AT) mantenidas en una plantación de Atriplex, NP-1,0) similar a AT más nopal (1,0\% del PV en BS) y NP-1,5) similar a AT más nopal $(1,5 \%$ del PV en BS). En el Cuadro 1 se resumen los experimentos y los tratamientos aplicados en cada uno.

Experimento 1: se utilizaron veinte cabras adultas de la raza Nubia de tres años de edad, lactantes (entre 2 y 2,5 meses posparto) y no preñadas. Previo al inicio del estudio, las cabras estaban en condiciones intensivas de producción, en estabulación y alimentación controlada. Diez de estas cabras, seleccionadas al azar, permanecieron en estabulación y alimentación controlada como control (CO), mientras las otras diez fueron asignadas al tratamiento experimental, que consistió en pastoreo en una plantación de Atriplex de las 9:00 a las 15:00 horas (AT). El resto del tiempo se alojaron en un corral común el cual tenía acceso al consumo de agua. La dieta ofrecida a las cabras del grupo control se calculó para cubrir las necesidades nutritivas de cabras de $45 \mathrm{~kg}$ con producciones de 2,5 1 diarios y estuvo compuesta por $47,5 \%$ de grano de sorgo, $10,0 \%$ de harina de soya, $40,0 \%$ de heno de alfalfa, $0,5 \%$ de minerales y $2,0 \%$ de sal común. Debido a que el objetivo de este experimento era determinar el potencial del forraje de Atriplex como principal fuente de alimento para cabras lactantes durante la sequía, no se incluyó un periodo de adaptación.

Experimento 2: se utilizaron las diez cabras previamente alimentadas con Atriplex en el Experimento 1. Estas se mantuvieron en pastoreo en 
Cuadro 1. Tratamientos utilizados en la alimentación de cabras, en tres experimentos. Soledad, San Luis Potosí, México. 2005.

\begin{tabular}{llll}
\hline & \multicolumn{1}{c}{ Trat. 1 } & \multicolumn{1}{c}{ Trat. 2 } & \multicolumn{1}{c}{ Trat. 3 } \\
\hline Experimento 1 & Estabulación y alimentación & Pastoreo en Atriplex & \\
& controlada & & \\
Experimento 2 & Pastoreo en Atriplex & $\begin{array}{l}\text { Pastoreo en Atriplex }+ \text { nopal } \\
(0,8 \% \text { del PV en BS })\end{array}$ & \\
& & Pastoreo en Atriplex + nopal & Pastoreo en Atriplex + nopal \\
Experimento 3 & Pastoreo en Atriplex & $(1,0 \%$ del PV en BS $)$ & $(1,5 \%$ del PV en BS $)$ \\
\hline
\end{tabular}

PV en BS: Peso vivo en base seca.

una plantación de Atriplex de las 8:30 a las 15:00 horas, permaneciendo en confinamiento el resto del día, donde dispusieron de agua fresca a libertad. Cinco de estas cabras fueron suplementadas con nopal en nivel de $0,8 \%$ del PV en BS (NP) y las otras cinco no recibieron suplementación (AT). El estudio tuvo una duración de seis semanas y no se consideró un periodo de adaptación, debido a que el efecto del consumo de nopal se reflejó desde la primera semana. Las pencas de nopal fueron cortadas diariamente de una plantación de cuatro años de edad, las cuales fueron picadas manualmente en pequeños trozos de aproximadamente $2 \times 5 \mathrm{~cm}$ y ofrecidas directamente a los animales en comederos comunes. La composición química promedio del nopal ofrecido se determinó tomando seis muestras al azar.

Experimento 3: se utilizaron catorce cabras adultas de la raza Nubia, lactantes (entre 2,5 y 3,5 meses posparto) y no preñadas, que se mantuvieron en pastoreo en una plantación de Atriplex de las 8:30 a las 15:00 horas, permaneciendo en confinamiento el resto del día, donde dispusieron de agua fresca a libertad. Cuatro de ellas fueron suplementadas con nopal en nivel de $1,0 \%$ del PV en BS (NP-1,0), otras cinco fueron suplementadas con nopal en nivel de $1,5 \%$ del PV en BS (NP-1,5) y las otras cinco no fueron suplementadas (AT). Se utilizaron dos niveles de suplementación de nopal bajo el supuesto de que a mayor consumo de carbohidratos solubles mejor aprovechamiento del $\mathrm{N}$ contenido en Atriplex, lo que se reflejaría en mejor respuesta animal. El estudio tuvo una duración de siete semanas, previo al cual las cabras se adaptaron al consumo de Atriplex y nopal por un periodo de 21 días. Durante este periodo, la cantidad de nopal ofrecido se incrementó gradualmente conforme se aumentó el consumo voluntario, al mismo tiempo que se redujo la cantidad de alimento integral (-100 g diarios). Para la tercera semana de adaptación, los individuos ya consumían el forraje pastoreado y el nopal. Este se les ofreció de manera similar a lo descrito en el Experimento 2. La composición química del nopal ofrecido fue: $\mathrm{MS}=10,06 \pm 1,2 \%, \quad \mathrm{PC}=4,42 \pm 0,32 \%, \quad \mathrm{FAD}=$ $16,6 \pm 2,31 \%, \mathrm{FND}=22,1 \pm 3,64 \%$, celulosa $=2,8 \pm 0,41 \%$, lignina $=2,1 \pm 0,17 \%$ y $E D=2,21 \pm 0,21 \mathrm{Mcal}$ of $\mathrm{DE} / \mathrm{kg}$.

Variables estudiadas. En los tres experimentos se registró cada siete días (desde el inicio del tratamiento), el peso corporal de las cabras en estado de ayuno. Además, la producción de leche se registró dos veces por semana (lunes y jueves), midiendo el volumen de leche producido, para lo cual se utilizó un vaso graduado con capacidad de $500 \mathrm{ml}$. Luego, se pesó en una báscula con una precisión de $0,1 \mathrm{~g}$. La densidad se estimó a partir del peso y el volumen obtenidos.

Análisis estadísticos. Los resultados de peso y producción de leche se compararon por medio de análisis de varianza con un modelo de medidas repetidas en el tiempo, incluyendo el tratamiento como efecto entre unidades experimentales y el tiempo como factor dentro de cada unidad experimental. La producción inicial se incluyó como covariable en el modelo. Los análisis se realizaron con el paquete estadístico JMP Star Statistics, Ver. 4.0.1. Academic (SAS Institute, 2000). 


\section{RESULTADOS Y DISCUSIÓN}

\section{Experimento 1}

La producción de leche disminuyó en ambos tratamientos durante las primeras cuatro semanas de pastoreo (Figura 1), detectándose diferencias debidas al tratamiento $(\mathrm{P}<0,08)$ y a la interacción entre el tratamiento y el periodo $(\mathrm{P}<0,0001)$. Durante este periodo la producción de leche disminuyó gradualmente (Cuadro 1 y Figura 1) en ambos grupos de cabras, siendo más acentuada la reducción en las cabras alimentadas con Atriplex. Durante el resto del estudio, la producción de leche continuó descendiendo, pero sólo en las cabras alimentadas exclusivamente con Atriplex, con diferencias debidas al tratamiento $(\mathrm{P}<0,0001)$, al periodo $(\mathrm{P}<0,0001)$ y a la interacción $(\mathrm{P}<0,05)$. Además, en las cabras alimentadas con Atriplex, la densidad de la leche se redujo significativamente $(\mathrm{P}<0,0001) \quad(1,10 \pm 0,03$ y $1,01 \pm 0,01$ en la leche de cabras confinadas y en Atriplex, respectivamente).

El forraje de Atriplex que estuvo disponible por cabra durante el experimento fue de $4,8 \mathrm{~kg} / \mathrm{d}$, conteniendo $17,2 \pm 2,85 \%$ de PC, $20,8 \pm 1,09 \%$ de FAD y $66,4 \pm 2,63 \%$ de DMS. De acuerdo con las tablas de requerimientos del National Research Council para cabras (NRC, 1981), la disponibilidad, junto con la calidad nutritiva que mostró el forraje de Atriplex

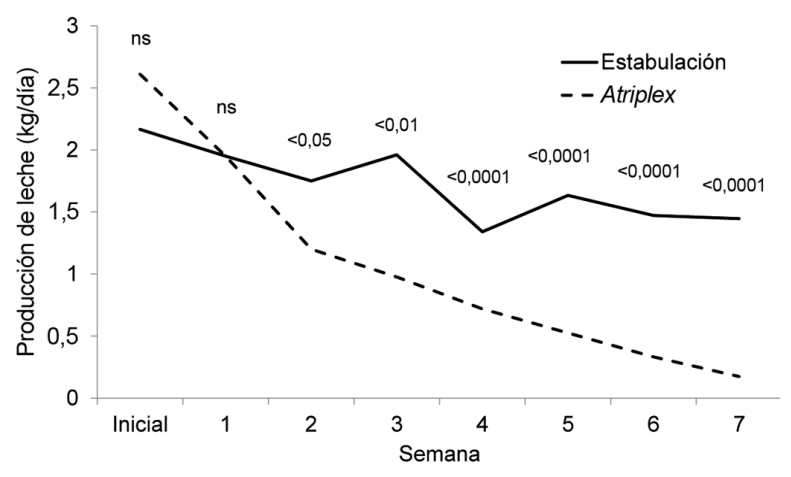

Figura 1. Producción de leche de cabras en estabulación y alimentación controlada o pastoreando en una plantación de Atriplex canescens durante un periodo de siete semanas en la estación seca del año. Soledad, San Luis Potosí, México. 20 de mayo al 8 de julio de 2005 . canescens fue suficiente para cubrir los requerimientos de materia seca de una cabra de $45 \mathrm{~kg}$ de peso, lo que sugiere que no hubo restricción en cuanto a la disponibilidad de materia seca y proteína se refiere. Esto se reflejó en el peso de las cabras, en el que no se detectaron diferencias $(P>0,05)$ entre tratamientos a lo largo del estudio (Cuadro 2). El elevado contenido de proteína cruda de Atriplex sugiere que el requerimiento de proteína fue cubierto.

La reducción observada en la producción de leche era de esperarse, pues el forraje de Atriplex, aunque contiene elevados niveles de proteína (Urrutia et al., 1989; Guerrero et al., 2010), por sí solo es incapaz de satisfacer los altos requerimientos de energía de las cabras con producciones de leche elevadas (Valderrábano et al., 1996; Abu-Zanat y Taba, 2004). Estos resultados indican que el forraje de Atriplex, como principal o única fuente de nutrientes, puede ser utilizado casi exclusivamente como dieta de mantenimiento.

\section{Experimento 2}

La producción inicial de leche fue similar en las cabras de los dos tratamientos (Cuadro 3 y Figura 2). La inclusión de nopal originó un incremento

Cuadro 2. Peso inicial y final, producción de leche inicial y final, y densidad de la leche de cabras mantenidas en confinamiento o pastoreando un banco de Atriplex. Soledad, San Luis Potosí, México. 20 de mayo al 8 de julio de 2005 .

\begin{tabular}{lcc}
\hline Variable & \multicolumn{2}{c}{ Tratamiento } \\
\cline { 2 - 3 } & Confinamiento & Atriplex \\
\hline $\begin{array}{l}\text { Peso vivo (todo el expe- } \\
\text { rimento) }\end{array}$ & - & - \\
$\quad$ Inicial & $43,0 \pm 4,6$ & $43,1 \pm 4,0$ \\
$\quad$ Final & $44,0 \pm 5,1$ & $43,1 \pm 4,5$ \\
\hline Producción de leche & & \\
$\quad$ Inicial & $2,17 \pm 0,71$ & $2,61 \pm 0,46$ \\
Final & $1,45 \pm 0,6 \mathrm{a}$ & $0,17 \pm 0,10 \mathrm{~b}$ \\
$\quad$ Promedio & $1,68 \pm 0,60 \mathrm{a}$ & $0,94 \pm 0,09 \mathrm{~b}$ \\
\hline Densidad de la leche & $1,10 \pm 0,03 \mathrm{a}$ & $1,01 \pm 0,01 \mathrm{~b}$ \\
\hline
\end{tabular}

Valores en la misma línea con distinta letra indica diferencias estadísticas $(\mathrm{P}<0,05)$. 
Cuadro 3. Peso inicial y final, producción de leche inicial y final, y densidad de la leche de cabras mantenidas en pastoreo en un banco de Atriplex con y sin suplementación con nopal $(0,8 \%$ del peso vivo en base seca). Soledad, San Luis Potosí, México. 20 de mayo al 8 de julio de 2005.

\begin{tabular}{lcc}
\hline & \multicolumn{2}{c}{ Tratamiento } \\
\cline { 2 - 3 } Variable & Atriplex & Atriplex + nopal \\
\hline Peso vivo & & \\
Inicial & $42,0 \pm 4,7$ & $46,1 \pm 3,7$ \\
Final & $37,2 \pm 2,4$ & $42,4 \pm 3,8$ \\
\hline Producción de leche & & \\
$\quad$ Inicial & $285,8 \pm 87,4$ & $288,0 \pm 103,8$ \\
Final & $152,1 \pm 78,2 \mathrm{a}$ & $306,2 \pm 65,7 \mathrm{~b}$ \\
$\quad$ Promedio & $170,3 \pm 51,8$ & $249,4 \pm 62,1$ \\
\hline Densidad de la leche & $1,02 \pm 0,05$ & $1,01 \pm 0,02$ \\
\hline
\end{tabular}

Valores en la misma línea con distinta letra indica diferencias estadísticas $(\mathrm{P}<0,05)$.

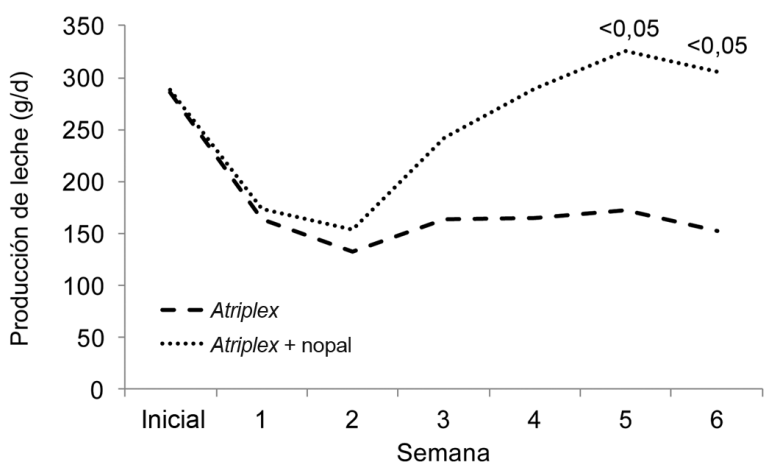

Figura 2. Producción de leche de cabras pastoreando en una plantación de Atriplex canescens más $0,8 \%$ de cladodios de nopal (\% del peso vivo en base seca) durante un periodo de seis semanas en la estación seca del año. Soledad, San Luis Potosí, México. 8 de julio al 19 de agosto de 2005.

significativo en la producción de leche, detectándose diferencias debidas al consumo de nopal $(\mathrm{P}<0,0001)$ y a la interacción entre el tratamiento y la semana de observación $(\mathrm{P}<0,02)$; sin embargo, no se encontró diferencias con respecto a la semana de observación $(\mathrm{P}>0,05)$. En las cabras que no recibieron nopal la producción de leche se mantuvo relativamente constante entre la segunda y última semana, mientras que en las que lo consumieron, la producción se incrementó hasta alcanzar alrededor de $300 \mathrm{~g} / \mathrm{d}$. Este incremento representó alrededor del doble de la producción obtenida en las cabras que no recibieron nopal. Sin embargo, la inclusión de nopal en la dieta de las cabras no originó cambios significativos $(\mathrm{P}>0,05)$ en la densidad de la leche $(1,02 \pm 0,05$ en las cabras alimentadas con Atriplex y $1,01 \pm 0,02$ con Atriplex + nopal).

El forraje disponible por cabra fue de $4,5 \mathrm{~kg} /$ d, conteniendo $16,7 \pm 2,36 \%$ de PC, 21,4 $4 \pm 1,69 \%$ de FAD y $65,3 \pm 2,45 \%$ de digestibilidad in situ de la materia seca. La composición media del nopal utilizado fue: $\mathrm{MS}=10,25 \pm 0,9 \%, \mathrm{PC}=4,69 \pm 0,27 \%$, $\mathrm{FAD}=17,4 \pm 2,56 \%$, fibra neutro detergente (FND)= $23,4 \pm 3,54 \%$, celulosa $=2,9 \pm 0,33 \%$, lignina $=2,2 \pm 0,21 \%$ y 2,22 $\pm 0,21$ Mcal de energía digestible (ED)/kg MS. A diferencia del primer experimento, el peso de las cabras en ambos grupos disminuyó significativamente en ambos grupos de cabras $(\mathrm{P}<0,05)$, aunque no se detectaron diferencias $(\mathrm{P}>0,05)$ entre tratamientos (Cuadro 3). La pérdida de peso mostrada por las cabras sugiere que el consumo de energía fue insuficiente para cubrir los elevados requerimientos de cabras lactantes, a pesar de que las producciones fueron inferiores a $300 \mathrm{~g}$ diarios. Las cabras suplementadas con nopal tendieron a perder menos peso $(8,2 \%$ del peso inicial) que la observada en las no suplementadas (11,3\%); adicionalmente, la producción final de leche fue casi el doble en las cabras suplementadas. En conjunto, estos resultados indican, por un lado, que el consumo de materia seca y de energía pudo ser mayor en las cabras suplementadas y, por otro, que la energía contenida en el nopal mejoró el balance nitrógeno:energía de las cabras, como se había sugerido anteriormente (Ben Salem et al., 2002c, 2004).

Se ha demostrado el papel complementario que tienen el Atriplex y el nopal en la alimentación de rumiantes (Nefzaoui y Ben Salem, 2002; Ben Salem et al., 2005). Mientras el forraje de Atriplex contiene altas cantidades de proteína (Urrutia et al., 1989; Guerrero et al., 2010), el contenido de energía es bajo (Valderrábano et al., 1996; Abu-Zanat y Taba, 2004). En contraste, las pencas de nopal contienen altas cantidades de carbohidratos solubles, aunque su contenido de N es bajo (Nefzaoui y Ben Salem, 2002). De esta forma, los resultados muestran que es posible alimentar con Atriplex a pequeños rumiantes, siempre que se suplemente con una fuente de energía, como 
es el caso del nopal, con el fin de mejorar la respuesta animal (Ben Salem et al., 2004, 2005).

\section{Experimento 3}

La producción de leche al inicio del estudio fue de $330,2 \pm 75,292,1 \pm 205$ y $276,5 \pm 66$ g/d en AT, NP1,0 y NP-1,5, respectivamente $(\mathrm{P}>0,05)$. Durante las dos primeras semanas del estudio, las cabras de los tres tratamientos redujeron su producción de leche, alcanzando alrededor de $150 \mathrm{~g} / \mathrm{d}$ al final de la segunda semana. A partir de este momento, la producción de las cabras del grupo control continuó descendiendo, mientras que la producción en los grupos que recibieron nopal se mantuvo relativamente constante, mostrando ligeros incrementos las cabras del grupo NP-1,5 (Figura 3). Conforme el experimento avanzó, las cabras del grupo AT redujeron su producción de tal forma que para la séptima semana la producción representó menos de la mitad de la producción obtenida en las cabras del NP-1,5 en el mismo periodo. De esta forma, las cabras del grupo AT mostraron una reducción del $75 \%$ de la producción inicial, siendo significativamente mayor $(\mathrm{P}<0,005)$ que las pérdidas observadas en los grupos NP-1,0 $(55,4 \%)$ y C-1,5 $(36,5 \%)$ (Cuadro 4).

El forraje de Atriplex que estuvo disponible por cabra durante el experimento fue de $3,87 \mathrm{~kg} / \mathrm{d}$, conteniendo $17,8 \pm 2,44 \%$ de PC, $20,2 \pm 1,91 \%$ de FAD y $67,1 \pm 3,65 \%$ de digestibilidad in situ de la materia seca. Las cabras de los tres tratamientos mantuvieron

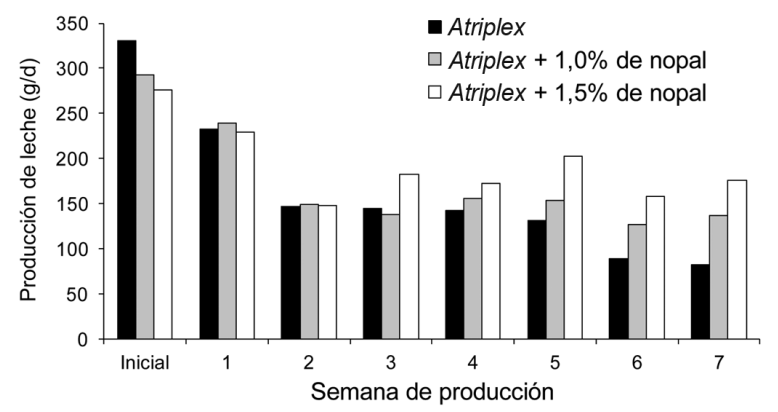

Figura 3. Producción de leche de cabras pastoreando en una plantación de Atriplex canescens más 1,0 y 1,5\% de cladodios de nopal (\% del peso vivo en base seca) durante un periodo de siete semanas en la estación seca del año. Soledad, San Luis Potosí, México. 16 de mayo al 27 de junio de 2006.

su peso corporal a lo largo del estudio. De acuerdo con los requerimientos sugeridos por el NRC (1981) para cabras, la disponibilidad, junto con la calidad nutritiva que mostró el forraje de Atriplex, fue suficiente para cubrir los requerimientos de materia seca y proteína cruda de una cabra de $45 \mathrm{~kg}$ de peso, lo cual sugiere que no hubo restricción en el consumo de estos nutrientes. Esto se reflejó en el peso de las cabras, las cuales mantuvieron su peso a lo largo del estudio (Cuadro 4).

Las cabras que no fueron suplementadas mantuvieron su peso corporal, pero con producciones de leche muy bajas, mientras que los animales que

Cuadro 4. Peso inicial y final, y producción de leche de cabras pastoreando en una plantación de Atriplex y suplementadas con 1,0 y 1,5\% de cladodios de nopal, durante siete semanas en la estación seca del año. Soledad, San Luis Potosí, México. 16 de mayo al 27 de junio de 2006.

\begin{tabular}{|c|c|c|c|c|c|}
\hline & \multicolumn{3}{|c|}{ Nivel de suplementación con nopal } & \multirow[t]{2}{*}{ E.E. } & \multirow[t]{2}{*}{ F calculada } \\
\hline & $\mathbf{0}$ & 1 & 1,5 & & \\
\hline Peso inicial (kg) & 37,8 & 35,1 & 38,0 & 1 & ns \\
\hline Peso final (kg) & 38,1 & 35,2 & 38,1 & 1,2 & ns \\
\hline Producción inicial de leche (g/d) & 330,2 & 292,1 & 276,5 & 31,1 & ns \\
\hline Producción final de leche (g/d) & 82 & 136,9 & 175,7 & 19 & ns \\
\hline Pérdida de producción de leche $(\%)$ & $75 c$ & $55,4 \mathrm{~b}$ & $36,5 \mathrm{a}$ & 6,8 & 0,005 \\
\hline Producción promedio de leche $(\mathrm{g} / \mathrm{d})^{*}$ & 162,4 & 178,7 & 193,1 & 22,5 & ns \\
\hline
\end{tabular}

* Producción promedio diaria del periodo de siete semanas. E.E.: Error estándar.

Valores en la misma línea con distinta letra indica diferencias estadísticas $(\mathrm{P}<0,05)$. ns: no significativo. 
consumieron nopal mantuvieron una producción de leche de alrededor del doble a la observada en las cabras alimentadas exclusivamente con Atriplex, lo que sugiere que la energía contenida en el nopal mejoró el balance energético de las cabras, reduciendo el nivel de pérdida de producción de leche (Figura 3 y Cuadro 4). Ciertamente, el nopal se ha considerado como fuente de energía para animales en condiciones semiáridas (Tegegne, 2002) y ha sido incorporado en los sistemas de alimentación basados en el pastoreo de arbustivas (Ben Salem et al., 2002 a,b). En estudios con ovinos se ha observado que la energía proporcionada por el nopal eleva la productividad (Ben Salem et al., 2004). En el presente estudio, las cabras mantuvieron su peso corporal, lo que sugiere que las mayores producciones de leche mostradas por las cabras suplementadas con nopal no fueron a expensas de sus reservas corporales, como había sido observado previamente (Chagra Dib et al., 2005; Ben Salem et al., 2010).

Las cabras que recibieron nopal (experimentos 2 y 3) mostraron una producción de leche de casi el doble de la producción registrada por aquellas que se alimentaron sólo con Atriplex, lo que sugiere que el consumo de nopal originó incrementos en el consumo total de materia orgánica digestible (Ben Salem et al., 2005; Ben Salem et al., 2010). Resultados similares se han observado en ovinos alimentados con dietas a base de pajas en los que el consumo de materia seca proveniente de paja se incrementa conforme se eleva la proporción de nopal en la dieta (Ben Salem et al., 1996).

En los experimentos 2 y 3, la inclusión de nopal evitó que los niveles de producción de leche se redujeran en la misma proporción que lo ocurrido en los grupos de cabras alimentadas exclusivamente con Atriplex. Estos resultados pueden atribuirse a la sincronía entre los carbohidratos solubles del nopal (Ben Salem et al., 1996) con el nitrógeno soluble del Atriplex (Ben Salem et al., 2002c; Ben Salem et al., 2010), lo cual no concuerda con los resultados obtenidos previamente con corderos en crecimiento alimentados con Atriplex (Ben Salem et al., 2005), en los que se observaron aumentos de peso cuando se incluyó nopal en la dieta.

Estos resultados sugieren la posibilidad de obtener entre 150 y $300 \mathrm{~g}$ diarios de leche manteniendo a las cabras en plantaciones de Atriplex incorporando nopal como fuente de energía a la dieta. En las regiones semiáridas de México, es común que la producción de leche se detenga durante la época de estiaje, e incluso, que las cabras experimenten pérdidas de peso. El nopal, un recurso abundante en estas regiones, representa una opción para evitar los estados recurrentes de restricción nutricional durante la sequía, abriendo la posibilidad de obtener una producción marginal de leche. Estos resultados son importantes para los caprinocultores de la región semiárida de México, donde las cabras podrían mantener una condición corporal moderadamente buena durante la sequía. Además, los productores estarían satisfechos por poder obtener una producción de 150 a $250 \mathrm{~g}$ diarios de leche durante esta época crítica del año sin recurrir al uso de alimentos concentrados, utilizando plantaciones de Atriplex en combinación con el uso de pencas de nopal como fuente de alimento.

\section{LITERATURA CITADA}

AOAC (Association of Official Analytical Chemistry). 2000. Official methods of analysis of the Association of Analytcal Chemists. 17 ed. Gaithersburg MD, USA.

Abu-Zanat, M.M.W., y M.J. Taba. 2004 Effect of feeding Atriplex browse to lactating ewes on milk yield and growth rate of their lambs. Small Rum. Res. 64:152161.

Beltrán, L.S. 1993. Análisis dimensional de costilla de vaca con fines predictivos. En: F. Rodríguez-Garza et al., editores, Memoria de la Reunión Nacional de Investigación Pecuaria. Jalisco 1993. INIFAP, Jalisco, Guadalajara, México.p. 2.

Ben Salem, H., H. Abdouli, A. Nefzaoui, A. El-Mastouri, y L. Ben-Salem. 2005. Nutritive value, behaviour, and growth of Barbarine lambs fed on oldman saltbush (Atriplex nummularia L.) and supplemented or not with barley grains or spineless cactus (Opuntia ficusindica f. inermis) pads. Special issue: Methodology, nutrition and products quality in grazing sheep and goats. Ed. Morand-Fehr, P; Ben Salem H; Papachristou T.G. Small Rum. Res. 59:229-237.

Ben Salem, H., A. Nefzaoui, H. Abdouli, y E.R. Ørskov. 1996. Effect of increasing level of spineless cactus (Opuntia ficusindica var. inermis) on intake and digestion by sheep fed straw-based diets. Anim. Sci. 62:293-299.

Ben Salem, H., A. Nefzaoui, y L. Ben Salem. 2002a. Supplementation of Acacia cyanophylla Lindl. 
foliage-based diets with barley or shrubs from arid areas (Opuntia ficus indica f. inermis and Atriplex nummularia L.) on growth and digestibility in lambs. Anim. Feed Sci. Technol. 96:15-30.

Ben Salem, H., A. Nefzaoui, y L. Ben Salem. 2002b. Nitrogen supplementation improves the nutritive value of Opuntia ficus-indica F. inermis-based diets and sheep growth. En: Acta Horticulturae, editor, IV International Congress on Cactus Pear and Cochineal. 31 August 2002. Hammamet, Tunisia. p. 317-321.

Ben Salem, H., A. Nefzaoui, y L. Ben Salem. 2002c. Opuntia ficus-indica $f$ inermis and Atriplex nummularia L.: Two complementary fodder shrubs for sheep and goats. En: Acta Horticulturae, editor, IV International Congress on Cactus Pear and Cochineal. 31 August 2002. Hammamet, Tunisia. p. 333-341.

Ben Salem, H., A. Nefzaoui, y L. Ben-Salem. 2004. Spineless cactus (Opuntia ficus-indica $f$. inermis) and oldman saltbush (Atriplex nummularia L) as alternative supplements for growing Barbarine lambs given straw-based diets. Small Rum. Res. 51:65-73.

Ben Salem, H., H.C. Norman, A. Nefzaoui, D.E. Mayberry, K.L. Pearce, y D.K. Revell. 2010. Potential use of oldman saltbush (Atriplex nummularia Lindl.) in sheep and goat feeding. Small Rum. Res. 91:13-28.

Castillo, C.M., G.E. Aparicio, y M.J. Urrutia. 1989. Caracterización de la caprinocultura en cinco ejidos del municipio de Venado, S.L.P. En: Asociación Mexicana de Producción Caprina A.C., editor, Memorias de la V Reunión Nacional sobre Caprinocultura. Zacataecas, Zacatecas, México. p. 28-31.

Chagra Dib, E.P., H.D. Leguiza, T. Vera, C. Valdivia, y O. Bazan. 2005. Utilización de Opuntia ficus indica y Atriplex nummularia en cabras criollas en lactancia. En: Asociación Latinoamericana de Producción Animal, editor, XIX Reunión de la Asociación Latinoamericana de Producción Animal. Tampico, Tamaulipas, México. BIOTAM Nueva serie. Edición especial. p. 261-264.

Echavarría, C.F.G., L.R. Gutiérrez, R.R.I. Ledesma, V.R. Bañuelos, S.J.I. Aguilera, y P.A. Serna. 2006. Influencia del sistema de pastoreo con pequeños rumiantes en un agostadero del semiárido Zacatecano. I Vegetación nativa. Téc. Pec. Méx. 44:203-217.

Guerrero, M., A.S. Juárez, R.G. Ramírez, R. Montoya, M. Murillo, O. La O, y M.A. Cerrillo. 2010. Composición química y degradabilidad de la proteína de forrajes nativos de la región semiárida del norte de México. Rev. Cubana Cienc. Agríc. 44:147-154.

Medina, G.G., P.G. Díaz, O.C. Loredo, A.V. Serrano, y G.M.A. Cano. 2005. Estadísticas climatológicas básicas del estado de San Luis Potosí (período 1961-2001). Libro Técnico No 2. Instituto Nacional de Investigaciones Forestales, Agrícolas y Pecuarias, Campo Experimental San Luis. San Luis Potosí, México.

Morlán, C.A.A., T.J. De Lucas, y L.E. Valdez. 2005. Caracterización de sistemas de producción de pequeños rumiantes en Venado y Villa de Arista, San Luis Potosí. En: Asociación Mexicana de Producción Caprina A.C., editor, Memorias de la XX Reunión Nacional sobre Caprinocultura. Culiacán, Sinaloa, México. p. 625-631.

Nefzaoui, A., y H. Ben Salem. 2002. Forage, fodder and animal nutrition. En: P.S. Nobel, editor, Cacti: biology and uses. University of California Press, Berkeley, California, USA. p. 190-210.

NRC (National Research Council). 1981. Nutrient requirements of goats: Angora, dairy, and meat goats in temperate and tropical countries. National Academy Press, Washington, D.C., U.S.A.

Olhagaray, C.O.R. 2005. Diagnóstico del segundo año del GGAVATT-INIFAP en el Ejido Juan E. García del municipio de Lerdo, Durango.En:Asociación Mexicana de Producción Caprina A.C., editor, Memorias de la XX Reunión Nacional sobre Caprinocultura. Culiacán, Sinalo., México. p. 615-624.

Ramírez, R.G., y M.A. Núñez-González. 2006. Chemical composition, digestion and mineral content of native forbs consumed by range sheep. J. Anim. Vet. Adv. 5:1158-1164.

SAS Institute. 2000. User's Guide: Statistics. Version 4.0.3 (Academic). SAS Inst., Cary, NC, USA.

Tegenge, F. 2002. Fodder potential of Opuntia ficus indica. En: Acta Horticulturae, editor, IV International Congress on Cactus Pear and Cochineal. 31 August 2002. Hammamet, Tunisia. p. 342-347.

Urrutia, M.J., C.A. Martínez, y D.J. Villanueva. 1989. Caracterización químico proximal de las plantas del genero Atriplex. En: Asociación Mexicana de Producción Caprina A.C., editor, V Reunión Nacional sobre Caprinocultura. Zacatecas, Zacatecas, México. p. 137-139. 
Valderrábano, J., F. Muñoz, y I. Delgado. 1996. Browsing ability and utilization by sheep and gotas of Atriplex halimus L. shrubs. Small Rumin. Res. 19:131-136.

Van Soest, P.J., J.B. Robertson, y B.A. Lewis. 1991. Methods for dietary neutral detergent fiber, and non starch polysaccharides in relation to animal nutrition. J. Dairy Sci. 74:3583-3589.

Vanzant, E.S., R.C. Cochran, y E.C. Titgemeyer. 1998. Standardization of in situ techniques for ruminant feedstuff evaluation. J. Anim. Sci. 76:2717-2729. 